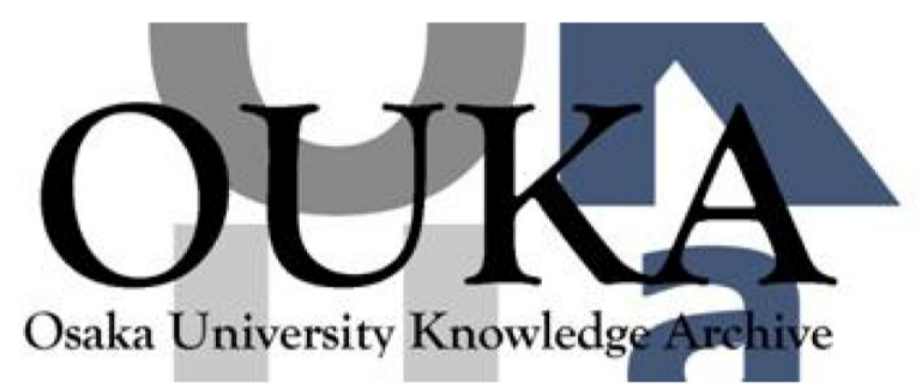

\begin{tabular}{|c|l|}
\hline Title & $\begin{array}{l}\text { Boson Number in Bose-Einstein Condensate of } \\
\text { Liquid Helium near the } \lambda \text { Point }\end{array}$ \\
\hline Author(s) & Sasaki, Shosuke \\
\hline Citation & $\begin{array}{l}\text { Journal of Low Temperature Physics. 148(3-4) } \\
\text { p. 103-p. 108 }\end{array}$ \\
\hline Issue Date & $2007-08$ \\
\hline oaire:version AM \\
\hline URL & https://hdl. handle. net/11094/27205 \\
\hline rights & \\
\hline Note & \\
\hline
\end{tabular}

Osaka University Knowledge Archive : OUKA

https://ir. Library. osaka-u. ac. jp/

Osaka University 


\title{
Boson Number in Bose-Einstein Condensate of Liquid Helium near the $\lambda$ Point
}

\author{
Shosuke Sasaki \\ Shizuoka Institute of Science and Technology 2200-2 Toyosawa Fukuroi, 437-8555, Japan
}

Bose-Einstein condensation has been well investigated in dilute atomic gases. For liquid helium system, the superfluid component is considered to be a background flow in the Landau theory. We study the relation between BoseEinstein condensate and superfluid component. The concept of dressed bosons is introduced, which are eigenstates of the total Hamiltonian. The total energy is the sum of the kinetic energy of the center of mass and Galilean invariant terms. Therefore, the energy of the dressed boson has a nonlinear form for their number distribution function. If it is required that the excitation energy of a dressed boson be in agreement with the elementary excitation energy of the Landau theory near $0.0 \mathrm{~K}$, then the functional form of the dressed boson energy can be determined. Because of this functional form, the dressed bosons with zero momentum have no friction against a vessel only in existence of a Bose-Einstein condensate. Consequently, the condensate of the dressed bosons with zero momentum is the superfluid component. The number $n_{0}$ of dressed bosons with zero momentum is calculated. It shows temperature dependence $\left(1-\left(T / T_{\lambda}\right)\right)^{1 / 3}$ near the $\lambda$ point, where $T$ is the temperature and $T_{\lambda}$ is the $\lambda$ transition temperature.

PACS numbers: 67.40.-w, 67.40.Db, 64.60.Fr.

\section{INTRODUCTION}

The superfluid component in a dilute atomic gas is well known to be a Bose-Einstein condensate. For liquid helium, the superfluid component is a background flow in the Landau theory. Therefore, it is necessary to investigate the entity of the background flow of liquid helium. The total Hamiltonian $H$ of liquid helium is $H=\sum_{\mathbf{p}} \frac{\mathbf{p}^{2}}{2 m} a_{\mathbf{p}}^{*} a_{\mathbf{p}}+\frac{1}{2 V} \sum_{\mathbf{p}, \mathbf{q}, \mathbf{k}} g(\mathbf{k}) a_{\mathbf{p}+\mathbf{k}}^{*} a_{\mathbf{q}-\mathbf{k}}^{*} a_{\mathbf{p}} a_{\mathbf{q}}$, where $m$ is the mass of a helium atom, $a_{\mathbf{p}}^{*}$ and $a_{\mathbf{p}}$ are the creation and 


\section{Shosuke Sasaki}

annihilation operators, and $g(\mathbf{k})$ is the interatomic potential. We introduce a unitary transformation $U$ from free states to eigen states of $H$. Then, all the eigenstates of $H$ are expressed using the transformation as $\mid$ eigenstate $>=U a_{\mathbf{p} 1}^{*} a_{\mathbf{p} 2}^{*} \cdots a_{\mathbf{p} N}^{*} \mid 0>$, where $\mid 0>$ is the vacuum state. A new creation operator is defined as $A_{\mathbf{p}}^{*}=U a_{\mathbf{p}}^{*} U^{-1}$, which indicates a " dressed boson operator" with an interaction cloud. We rewrite the eigenstate using the dressed boson operators, thereby obtaining the following.

$$
\text { |eigenstate }>=A_{\mathbf{p}_{1}}^{*} A_{\mathbf{p}_{2}}^{*} \cdots A_{\mathbf{p}_{N}}^{*} U\left|0>=A_{\mathbf{p}_{1}}^{*} A_{\mathbf{p}_{2}}^{*} \cdots A_{\mathbf{p}_{N}}^{*}\right| 0>
$$

In that equation, we have used the equation $U|0>=| 0>$ because the vacuum state $\mid 0>$ is an eigenstate of $H$. Thus, all the eigenstates of liquid helium are expressed by direct products of the dressed boson operators. In this article, we express the total energy of liquid helium by dressed boson number operators $\left(n_{\mathbf{p}}=A_{\mathbf{p}}^{*} A_{\mathbf{p}}\right)$. The total energy has a non-linear form of the dressed boson number operators with Galilean invariant terms. BoseEinstein condensation of the dressed bosons occurs below the $\lambda$ point. This condensate of the dressed bosons is proven to be superfluid component, and $n_{0} / N$ is also proven to be proportional to $\left(1-\left(T / T_{\lambda}\right)\right)^{1 / 3}$ near the $\lambda$ point. In the next paper, we will clarify the appearing of logarithmic divergence in the specific heat at the $\lambda$ point using the formulation of this article.

\section{TOTAL ENERGY OF DRESSED BOSONS}

The eigenenergy $E$ is expressed with the number distribution $\left\{n_{\mathbf{p}}\right\}$ of the dressed bosons in momentum space: $E=E\left(\left\{n_{\mathbf{p}}\right\}\right)$. The total Hamiltonian $H$ is the Galilean covariant, and the diagonal form of $H$ is the sum of the kinetic energy $K$ of the center of mass and Galilean invariant terms $X$.

$$
K=\frac{1}{2 M} \sum_{\mathbf{p}} \mathbf{p} n_{\mathbf{p}} \cdot \sum_{\mathbf{q}} \mathbf{q} n_{\mathbf{q}}=-\frac{1}{2 M} \sum_{\mathbf{p}, \mathbf{q}} \frac{1}{2}(\mathbf{p}-\mathbf{q})^{2} n_{\mathbf{p}} n_{\mathbf{q}}+\sum_{\mathbf{p}} \frac{\mathbf{p}^{2}}{2 m} n_{\mathbf{p}}
$$

In the equation above, $N=\sum_{\mathbf{q}} a_{\mathbf{q}}^{*} a_{\mathbf{q}}=\sum_{\mathbf{q}} A_{\mathbf{q}}^{*} A_{\mathbf{q}}=\sum_{\mathbf{q}} n_{\mathbf{q}}$. Also, $M$ is the total mass and $m=M / N$ is the mass of helium atom. Then, the diagonal form of the total Hamiltonian $H$ is expressed as the following.

$$
\begin{aligned}
H=K+X=\sum_{\mathbf{p}} \frac{\mathbf{p}^{2}}{2 m} n_{\mathbf{p}}-\frac{1}{2 M} \sum_{\mathbf{p}, \mathbf{q}} \frac{1}{2}(\mathbf{p}-\mathbf{q})^{2} n_{\mathbf{p}} n_{\mathbf{q}}+X \\
=\sum_{\mathbf{p}} \frac{\mathbf{p}^{2}}{2 m} n_{\mathbf{p}}+(\text { Galilean invariant terms })
\end{aligned}
$$

Therein, the Galilean invariant terms are described only by relative momenta of dressed bosons. Consequently, we can express the total energy using an 


\section{Boson Number in Bose-Einstein Condensate of Liquid Helium II}

arbitrary function $f$ as

$$
E=\sum_{\mathbf{p}} \frac{\mathbf{p}^{2}}{2 m} n_{\mathbf{p}}+\frac{1}{N} \sum_{\mathbf{p}, \mathbf{q}} f(\mathbf{p}-\mathbf{q}) n_{\mathbf{p}} n_{\mathbf{q}}
$$

The Galilean invariant term is determined such that the energy spectrum is in agreement with the Landau theory at $0.0 \mathrm{~K}$.

The ground state energy is $E_{G}=f(0) N$ when all the dressed bosons have momentum zero $\left(n_{0}=N\right)$. Therefore, $-f(0)$ is the latent heat per atom at $0.0 \mathrm{~K}$. The first excited energy is obtained for the number distribution of dressed bosons $\left\{n_{0}=N-1, n_{\mathbf{p}}=1\right\}$ :

$$
\begin{aligned}
E=\frac{\mathbf{p}^{2}}{2 m}+ & \frac{1}{N}\left[f(0)(N-1)^{2}+f(\mathbf{p})(N-1)+\right. \\
& +f(-\mathbf{p})(N-1)+f(\mathbf{p}-\mathbf{p})] \approx f(0) N+\frac{\mathbf{p}^{2}}{2 m}+2(f(\mathbf{p})-f(0)) .
\end{aligned}
$$

Therein, we have used $1 / N \approx 0$ and the spherical symmetric property of the function $f(\mathbf{p})$. If we take the functional form as $f(\mathbf{p})=\frac{1}{2}\left(\epsilon_{\mathbf{p}}^{0}-\left(\mathbf{p}^{2} /(2 m)\right)+\right.$ $f(0)$, where $\epsilon_{\mathbf{p}}^{0}$ is the elementary excitation energy at $0.0 \mathrm{~K}$, then we obtain the first excited energy of the total system as $E=E_{G}+\epsilon_{\mathbf{p}}^{0}$. This excitation energy $E-E_{G}$ is in agreement with the Landau theory. It is noteworthy here that the functional form of $f(\mathbf{p})$ has been determined from experimental data of the elementary excitation energy $\epsilon_{\mathbf{p}}^{0}$ and the latent heat at the temperature $T=0$. Therefore, the present theory is equivalent to the Landau theory near $0.0 \mathrm{~K}$. The excitation energy of dressed boson depends upon $T$, when the temperature becomes high. The energy of the dressed boson is the energy increase when a single dressed boson is added to the system, as

$$
\omega_{\mathbf{p}}=\frac{\delta E}{\delta n_{\mathbf{p}}}=\frac{\mathbf{p}^{2}}{2 m}+\frac{2}{N} \sum_{\mathbf{q}} f(\mathbf{p}-\mathbf{q}) n_{\mathbf{q}}-\frac{1}{N^{2}} \sum_{\mathbf{s}, \mathbf{t}} f(\mathbf{s}-\mathbf{t}) n_{\mathbf{s}} n_{\mathbf{t}},
$$

where we have used $f(\mathbf{p}-\mathbf{q})=f(\mathbf{q}-\mathbf{p})$. The distribution function of dressed boson number is given as $n_{\mathbf{p}}=1 /\left[\exp \left(\left(\omega_{\mathbf{p}}-\mu\right) /\left(k_{B} T\right)\right)-1\right]$, where $\mu$ is the chemical potential and $k_{B}$ is the Boltzmann constant. The chemical potential $\mu$ is nearly equal to $\omega_{0}$ for $T<T_{\lambda}$. That value means that $n_{0}$ is a macroscopic number for $T<T_{\lambda}$. Therefore, Bose-Einstein condensation of the dressed bosons occurs. The excitation energy from the Bose condensate is $\epsilon_{\mathbf{p}}=\omega_{\mathbf{p}}-\omega_{0}=\frac{\mathbf{p}^{2}}{2 m}+\frac{2}{N} \sum_{\mathbf{q}} f(\mathbf{p}-\mathbf{q}) n_{\mathbf{q}}$. Hereafter, we call this excitation from the Bose-Einstein condensate BEC excitation. This BEC excitation energy agrees with Landau excitation energy near $T=0$ and varies according to temperature change. We obtain the functional form for a small momentum $p$ as shown below.

$$
\omega_{\mathbf{p}}-\omega_{0}=\frac{n_{0}}{N} c p+\operatorname{Order}\left(p^{2}\right)
$$




\section{Shosuke Sasaki}

The equation above is derived from dividing the summation in (3) to two regions as $q<q_{A}$ and $q>q_{A}$ :

$$
\begin{array}{r}
\omega_{\mathbf{p}}=\frac{\mathbf{p}^{2}}{2 m}+\frac{n_{0}}{N} c|\mathbf{p}|+\frac{V}{N(2 \pi \hbar)^{3}} \int_{q=0}^{q_{A}} \iint c|\mathbf{p}-\mathbf{q}| n_{\mathbf{q}} \mathrm{d} \cos \theta \mathrm{d} \phi q^{2} \mathrm{~d} q+ \\
+\frac{2 V}{N(2 \pi \hbar)^{3}} \int_{q=q_{A}}^{\infty} \iint f(\mathbf{p}-\mathbf{q}) n_{\mathbf{q}} \mathrm{d} \cos \theta \mathrm{d} \phi q^{2} \mathrm{~d} q+(\text { constant value }), \\
\frac{V}{N(2 \pi \hbar)^{3}} \int_{0}^{q_{A}} \iint c|\mathbf{p}-\mathbf{q}| n_{\mathbf{q}} \mathrm{d} \cos \theta \mathrm{d} \phi q^{2} \mathrm{~d} q=\frac{2 \pi V}{N(2 \pi \hbar)^{3}} \int_{0}^{q_{A}} \int_{-1}^{1} c \sqrt{p^{2}-2 p q t+q^{2}} \\
\times \mathrm{d} t n_{q} q^{2} \mathrm{~d} q=\frac{2 \pi V}{N(2 \pi \hbar)^{3}} \int_{0}^{q_{A}} c \frac{|p+q|^{3}-|p-q|^{3}}{3 p q} n_{q} q^{2} \mathrm{~d} q=\operatorname{Order}\left(p^{2}\right)+(\text { constant value }) \\
\frac{2 V}{N(2 \pi \hbar)^{3}} \int_{q_{A}}^{\infty} \iint f(\mathbf{p}-\mathbf{q}) n_{\mathbf{q}} \mathrm{d} \cos \theta \mathrm{d} \phi q^{2} \mathrm{~d} q=\frac{2 V}{N(2 \pi \hbar)^{3}} \int_{q_{A}}^{\infty} \iint[f(-\mathbf{q})+ \\
\left.+\alpha \mathbf{p} \cdot \mathbf{q}+\beta p^{2}+\cdots\right] n_{\mathbf{q}} \mathrm{d} \cos \theta \mathrm{d} \phi q^{2} \mathrm{~d} q=\operatorname{Order}\left(p^{2}\right)+(\text { constant value }) .
\end{array}
$$

Therein, we have used $f(\mathbf{p}-\mathbf{q})=\frac{1}{2}\left(\epsilon_{\mathbf{p}-\mathbf{q}}^{0}-(\mathbf{p}-\mathbf{q})^{2} /(2 m)\right)+f(0)=\frac{1}{2} c|\mathbf{p}-\mathbf{q}|+$ $f(0)+\operatorname{Order}(\mathbf{p}-\mathbf{q})^{2}$ for small momenta $p<q_{A}$ and $q<q_{A}$, because $\epsilon_{\mathbf{p}}^{0}=c|\mathbf{p}|$ for a small value of $p$. As shown in Eq.(4), the velocity of a dressed boson becomes $n_{0} c / N$ which approaches zero at the $\lambda$ point because $n_{0} / N \rightarrow 0$ for $T \rightarrow T_{\lambda}$ [see ref. 1]. This property of BEC excitation is similar to that of the second sound mode in Brillouin scatterings $[2,3,4]$, where the velocity of the second sound becomes zero at the $\lambda$ point. The second sound mode is considered to be a thermal wave mode and its theoretical width becomes infinitely large at the $\lambda$ point in the traditional theory. However, the experimental width is less than $1 / 10$ of the first sound width at temperature $T=T_{\lambda}-0.5 \mathrm{mK}$ [see ref. 4]. Therefore, the lifetime of the second sound mode is longer than about 10 times of the first sound mode. This evidence implies that difficulties exist in the traditional interpretation. For that reason, we adopt a novel viewpoint that the second sound mode detected in Brillouin scattering near the $\lambda$ point is the BEC excitation.

\section{CONDENSED DRESSED BOSON NUMBER}

Bendt et al. [5], and Brooks and Donnelly [6] (which is called BD theory hereafter) calculated thermodynamic functions on the basis of Landau theory using temperature dependence of excitation energy. In this paper, we calculate the BEC excitation number $N_{E}$, defined as the following.

$$
N_{E}=\frac{V}{(2 \pi \hbar)^{3}} \int_{0(p \neq 0)}^{\infty} n_{p} 4 \pi p^{2} \mathrm{~d} p
$$




\section{Boson Number in Bose-Einstein Condensate of Liquid Helium II}

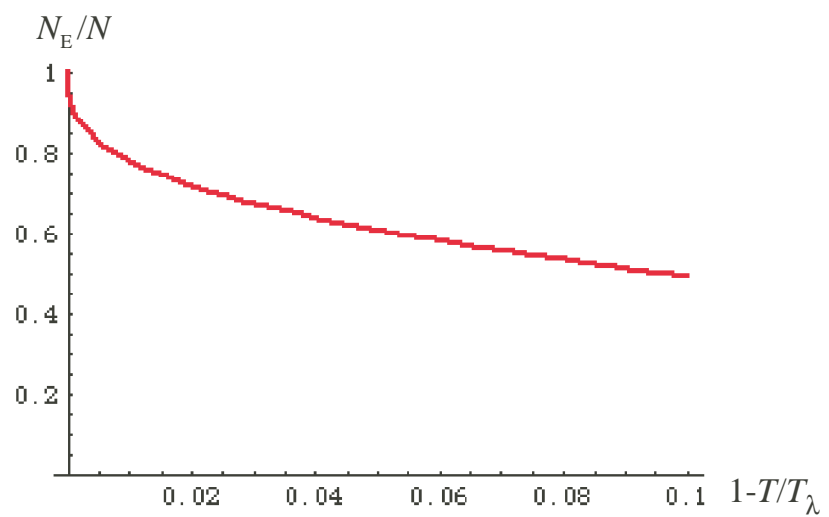

Fig. 1. The curve indicates $N_{E} / N$.

For that definition, $n_{p}=\left(\exp \left[\left(\omega_{p}-\mu\right) /\left(k_{B} T\right)\right]-1\right)^{-1}$. Therin, $\mu$ is the chemical potential. As in the BD theory [6], the integral region is divided into four regions of phonon, maxon, roton, and higher momentum. For the phonon region, we adopt the BEC excitation energy as

$\omega_{p}-\omega_{0}=p c_{2}\left(1-\left(T / T_{\lambda}\right)+a p^{3}\right)^{1 / 3}+p D\left(1-\left(T / T_{\lambda}\right)+b p^{2}\right)^{1 / 2}\left(\right.$ for $\left.0<p<q_{A}\right)$,

which has the second sound velocity of reference [7] for a small $p$, and is proportional to $p^{2}$ for a large $p$. Coefficients $c_{2}$ and $D$ are determined from the second sound velocity [7]; $a, b$ are parameters.

The function parameters in the maxon region are determined such that the excitation energy and its tangent are continuously connected to the two neighbor curves. We use the functional forms for the roton and higher momentum regions, just as in the $\mathrm{BD}$ theory. Then, the temperature dependence of $N_{E} / N$ is evaluated: the result is shown in Fig. 1. They show that $N_{E}$ becomes $100 \%$ of the total number of helium atoms at the $\lambda$ point. The number $n_{0}$ of dressed-bosons in a Bose-Einstein condensate is given as $n_{0}=N-N_{E}$. The functional form of $\left(1-\left(T / T_{\lambda}\right)\right)^{1 / 3}$ well fits the calculated value of $n_{0} / N$ as in Fig.2. The critical exponent of $n_{0} / N$ is equal to $1 / 3$.

This calculated result, namely $n_{0} / N \propto\left(1-\left(T / T_{\lambda}\right)\right)^{1 / 3}$, indicates that the velocity of dressed boson is proportional to $\left(1-\left(T / T_{\lambda}\right)\right)^{1 / 3}$, as derived from Eq.(4). This result reproduces that the second sound velocity is proportional to $\left(1-\left(T / T_{\lambda}\right)\right)^{1 / 3}$ near the $\lambda$ point.

We consider a collision of a dressed boson with zero momentum against a macroscopic body. When the dressed boson receives momentum transfer $p$, energy-momentum conservation produces the following equation.

$$
\omega_{p}-\omega_{0}=\mathbf{p} \cdot \mathbf{v}
$$




\section{Shosuke Sasaki}

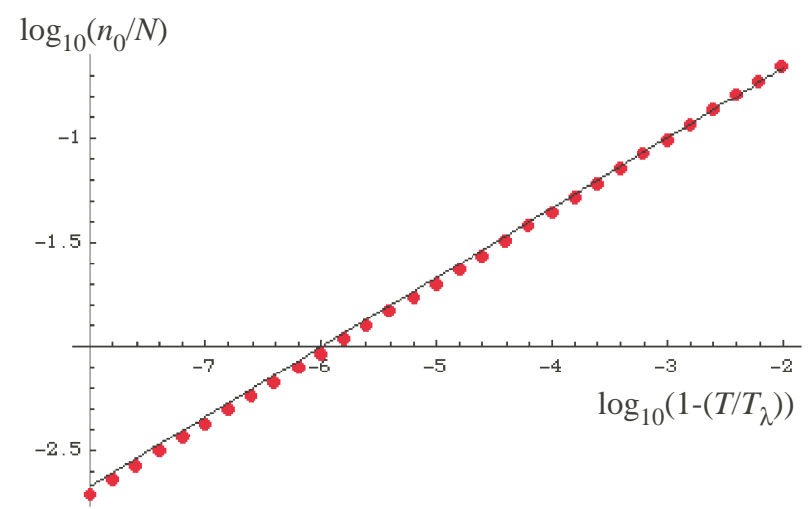

Fig. 2. The line indicates $\log _{10}\left(1-\left(T / T_{\lambda}\right)\right)^{1 / 3}$ and the dots are calculated values of $\log _{10}\left(n_{0} / N\right)$.

Therein, $\mathbf{v}$ is the velocity of the macroscopic body. If the magnitude of $\mathbf{v}$ is smaller than the critical velocity, then Eq. (7) does not hold for any momentum p. Consequently, the Bose-condensed dressed bosons receive no friction from the macroscopic body. That is to say, Bose-Einstein condensate of dressed bosons is superfluid component of liquid helium. Accordingly, it seems that the background flow in Landau theory is Bose-Einstein condensate of dressed bosons.

The value $n_{0}$ indicates the expectation value $<A_{0}^{*} A_{0}>$ of the dressed boson number with zero momentum which is different from $\left.<a_{0}^{*} a_{0}\right\rangle$. The value of $\left\langle a_{0}^{*} a_{0}\right\rangle$ is the expectation value of the number of helium atoms with zero momentum, which is measured in the neutron scattering and is a few percents at $T=0$. On the other hand, $\frac{n_{0}}{N}=\frac{\left\langle A_{0}^{*} A_{0}>\right.}{N}$ is the ratio of the superfluid component in the total liquid helium. This value becomes $100 \%$ at zero temperature.

\section{REFERENCES}

1. S. Sasaki, Springer Series in Solid State Science 79, 160 (1989).

2. G. Winterling, F. S. Holmes, and T. J. Greytak, Phys. Rev. Lett. 30, 427 (1973). G. Winterling, J. Miller, and T. J. Greytak, Phys. Lett. 48A, 343 (1974).

3. W. F. Vinen, C. J. Palin and J. M. Vaughan, Proc. of 13th Conf. on Low Temperature Physics 524 (1972).

4. J. M. Vaughan, W. F. Vinen and C. J. Palin, Proc. of 13th Conf. on Low Temperature Physics 532 (1972).

5. J. Bendt, R. D. Cowan, and J. L. Yarnell, Phys. Rev. 113, 1386 (1959).

6. J. S. Brooks and R. J. Donnelly, J. Phys. Chem. Ref. Data 6, 51 (1977).

7. D. S. Greywall and G. Ahlers, Phys. Rev. A 7, 2145 (1973). 